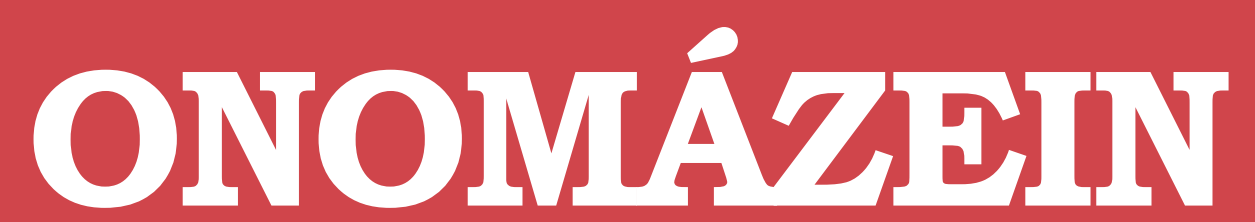

Revista semestral de lingüística, filología y traducción
PONTIFICIA UNIVERSIDAD CATÓLICA DE CHILE CATOLICA DE CHILE
FACULTAD DE LETRAS

\title{
La evidencialidad en la construcción de la significación histórica por estudiantes de Licenciatura de Historia ${ }^{1}$
}

Evidentiality in the construction of historical

significance by History majors

\section{Teresa Oteíza}

Pontificia Universidad

Católica de Chile

\section{Mabelin Garrido}

Pontificia Universidad

Católica de Chile

\section{Constanza Dalla Porta}

Pontificia Universidad

Católica de Chile

ONOMÁZEIN Número Especial IX ALSFAL (2014): 57-80

DOl: 10.7764/onomazein.alsfal.5

\section{(c) $\underset{\mathrm{BY}}{ } \bigodot_{\mathrm{ND}}$}

Teresa Oteíza: Facultad de Letras. Pontificia Universidad Católica de Chile. Correo electrónico: moteizas@uc.cl

Constanza Dalla Porta : Pontificia Universidad Católica de Chile. Correo electrónico: cadalla@uc.cl Mabelin Garrido : Pontificia Universidad Católica de Chile. Correo electrónico: maydani@gmail.com 


\section{Resumen}

Este artículo examina los mecanismos histórico-discursivos para la construcción de la evidencialidad como parte de las explicaciones históricas utilizadas por estudiantes de Licenciatura en Historia. El corpus de la investigación lo componen 16 respuestas escritas de controles de lectura de estudiantes de primer a tercer año de la carrera de Licenciatura en Historia de diferentes cursos de historia de Chile. La metodología de investigación posee un enfoque interdisciplinario que combina teorías y métodos de alfabetización histórica y de la Lingüística Sistémico Funcional, particularmente el modelo de valoración (White, 2003; Martin y White, 2005; Martin y Rose, 2007). El artículo desarroIla las posibilidades metodológicas del sistema de compromiso con el objeto de identificar la fuente de los significados interpersonales utilizados para incorporar evidencialidad en el discurso. Los resultados de la investigación presentados en el artículo abordan los factores que inciden en el comportamiento de la evidencialidad, como la formulación de la pregunta del examen y el tipo de evidencia (fuente primaria y bibliografía secundaria). Los resultados arrojan tres tendencias predominantes en los textos: a) la utilización estratégica de heteroglosia por intravocalización de contracción dialógica, la cual es usada como manera de indicar al evaluador el conocimiento de los hechos presentados en la fuente primaria, pero sin generar una explicación más elaborada de significación histórica; b) la utilización de heteroglosia por extravocalización de Atribución de Asimilación, sin que esto implique que la incorporación de la evidencia sea de buena calidad, ya que en muchos casos se incorpora la evidencia de manera errónea y/o estratégica de los hechos; c) la utilización de recursos de heteroglosia de contracción dialógica y de monoglosia que encubren la voz de la evidencia.

Palabras clave: alfabetización académica; evidencialidad; significación histórica; modelo de valoración; sistema de compromiso.

\section{Abstract}

This article examines the historical and discursive mechanisms to construct evidentiality as part of the historical explanations used by History majors in written texts. The corpus of the research is composed of 16 written answers obtained from the reading tests taken by first to third year history majors from three different courses of History of Chile. The research methodology takes an interdisciplinary approach that combines theories and methods from historical literacy and Systemic Functional Linguistics, particularly the appraisal framework (White, 2003; Martin \& White, 2005; Martin \& Rose, 2007). The article develops the methodological possibilities of the system of EN-
GAGEMENT with the purpose of identifying the source of interpersonal meanings used to incorporate evidentiality in the discourse. The results of the research presented in the article take into account the implicated factors by which evidentiality works, as well as the formulation of the test's questions and the type of evidence (primary source and secondary bibliography). The results show three predominant tendencies in the texts: a) the use of strategic heterogloss of intervocalization of dialogic contraction, which is used as a way to indicate the evaluator the knowledge of the facts presented in the primary source, without a more elaborated explanation of historical significan-

1 Este artículo es producto del Proyecto Interdisciplinario Vicerrectoría de Investigación, PUC, 2011-2013, Facultad de Educación, Facultad de Letras e Instituto de Historia: "El camino de Clío: la adquisición y el desarrollo de las habilidades de pensamiento histórico en producciones escritas de los estudiantes de Licenciatura en Historia de la Pontificia Universidad Católica de Chile". 
ce; b) the use of heterogloss of extravocalization of Attribution by Assimilation, without the implication that this incorporation of the evidence is of good quality, due to the fact that, in many cases, the evidence of the facts are incorporated in either a wrong or a strategic manner; and c) the use of heteroglossic resources of dialogic contraction and monoglossic resources that cover the voice of the evidence.

Keywords: academic literacy; evidentiality; historical significance; appraisal framework; ENGAGEMENT system.

\section{Introducción}

Este trabajo se inserta en el ámbito de la alfabetización académica y, de manera más particular, en la alfabetización académica de la historia. Es reconocido por muchos investigadores en la actualidad que las disciplinas construyen un tipo de conocimiento a través del lenguaje que posee características particulares (Bernstein, 2000; Martin y Rose, 2008; Maton y otros, 2010; Maton, 2014). De este modo, si bien el discurso académico comparte rasgos discursivos y lexicogramaticales en general, se ha documentado ampliamente que las diferentes disciplinas poseen sus modos particulares de significación y, por lo mismo, de construcción discursiva.

El discurso de la historia disciplinar y pedagógica ha sido estudiado en diferentes idiomas y en contextos educativos (Achugar, 2011; Coffin, 2006; Manghi, 2013a y b; Giudice y Moyano, 2011, 2013; Martin, 2002; Martin y Rose, 2008; Martin y Wodak, 2003; Moss, 2010; Moss y otros, 2013; Moyano, 2010; Oteíza, 2006, 2009, 2011, 2013; Oteíza y Pinuer, 2012, 2013; Pinto, 2011; Schleppegrell, 2004; Schleppegrell y otros, 2004; Veel y Coffin, 1996, entre muchos otros), lo que nos permite caracterizar lingüísticamente este ámbito de registro académico y su instanciación en una variedad de géneros históricos en contextos de educación secundaria y universitaria. De esta manera, los discursos históricos comparten con otros discursos de registro académico del área de las humanidades un lenguaje especializado con características lingüísticas prototípicas como la fuerte nominalización - que permite en parte la construcción de la abstracción y que se traduce en un empaquetamiento o 'cosificación' de los procesos y del tiempo-, así como la tendencia a la objetivación y la existencialización de los eventos y el tratamiento impersonal de las relaciones causales que tienden a realizarse al interior de las cláusulas.

Sin embargo, consideramos que el trabajo de alfabetización recién comienza en relación a la comprensión de cómo se construye la significación histórica desde el tratamiento de la evidencialidad, esto es, de qué manera los estudiantes de Historia manejan la construcción de patrones de evidencia a partir de las fuentes primarias y de la bibliografía secundaria que deben leer para sus cursos, para elaborar, a partir de ellas, argumentaciones históricas en sus escritos académicos. Este es un proceso complejo que debe enfrentar todo/a historiador/a y, por tanto, nos interesó analizar los mecanismos históricos y discursivos que activan los estudiantes universitarios de Licenciatura en Historia para construir evidencialidad como parte de las explicaciones históricas en diferentes momentos de su formación académica.

Consideramos que esta investigación, si bien se ha realizado en un corpus reducido, puede colaborar en nuestra comprensión de cómo se forma un/a historiador/a, cómo va aprendiendo a desarrollar un pensamiento histórico y de qué manera se puede generar un proceso que colabore con el aprendizaje de construcción de significación histórica y de la argumentación en la disciplina. Pensamos, asimismo, que este tipo de formación es vital no sólo para quienes deseen seguir la ruta de historiadores/as investigadores/as, sino que es igualmente importante para quienes luego decidan ser profesores/as 
de historia de enseñanza secundaria en el país y se vean enfrentados/as a la compleja tarea de ayudar a sus estudiantes a producir en esta área disciplinar.

De esta manera, el objetivo general de la investigación fue identificar las principales características y condiciones bajo las que se estructura el modo de razonamiento histórico de los estudiantes que cursan la carrera de Licenciatura en Historia en la Pontificia Universidad Católica de Chile (PUC). Específicamente, en este trabajo nos proponemos analizar los mecanismos discursivos de incorporación de la evidencia histórica a través de producciones escritas de estudiantes en controles de lectura, dado que la forma en la que se incluye la evidencia es crítica en la construcción de las cadenas causales que configuran las explicaciones históricas de los estudiantes. El corpus de la investigación lo componen 16 respuestas escritas de estudiantes de primer a tercer año de la carrera de Licenciatura en Historia de la PUC de tres cursos de historia de Chile que abarcan desde el siglo XV al siglo XIX.

La metodología de investigación se enmarca en un paradigma de análisis cualitativo y de carácter interdisciplinario. Se trabajó con teorías y métodos de alfabetización histórica con el objetivo de comprender el papel del lenguaje en su construcción (Paxton, 2002; Stahl y Shanahan, 2004) y el análisis de la evidencia (Henríquez y Ruiz, 2014; Oteíza, 2014) para identificar los factores explicativos presentes en los textos evaluados (fuentes primarias y bibliografía secundaria). Asimismo, el análisis del discurso se abordó desde la Lingüística Sistémico Funcional (LSF) y, particularmente, desde el modelo de valoración (White, 2003; Martin y White, 2005; Martin y Rose, 2007). El artículo desarrolla las posibilidades metodológicas del modelo de valoración para el análisis del discurso histórico de estudiantes de nivel universitario.

Este artículo se organiza de la siguiente ma- nera: en la segunda sección, presentamos sucintamente los marcos teóricos y metodológicos que guían nuestro análisis, esto es, el sistema de compromiso del modelo de la valoración inserto en el paradigma de la LSF, y la consideración del mismo como marco de análisis de la intertextualidad. Este, a su vez, nos permite abordar el tratamiento de la dimensión de evidencialidad y de alfabetización histórica. A continuación, la sección de análisis y discusión se refiere a la presentación del corpus a través de ejemplos representativos, y el análisis del mismo. Por último, presentamos una sección de conclusiones con los resultados de la investigación en relación a los factores que inciden en el comportamiento de la evidencialidad como la formulación de la pregunta del control escrito y el tipo de evidencia (fuente primaria y bibliografía secundaria) que se le proporciona al estudiante.

\section{Marco analítico}

Así como se ha planteado en la introducción de este trabajo, nuestro objetivo principal es determinar los mecanismos de construcción de la dimensión de evidencialidad de los estudiantes de Historia en la formulación de respuestas escritas en controles de lectura. Esto implica analizar las formas en que incluyen la información contenida en las fuentes primarias y bibliografías secundarias que han sido asignadas como lecturas obligatorias por los profesores respectivos de los cursos de Historia de Chile y América en los tres primeros años de su carrera universitaria. De este modo, consideramos fundamental para examinar el proceso de construcción de significación histórica el análisis de los mecanismos para incorporar hechos, condiciones y motivaciones de manera aislada o integrados en planteamientos, ideas nucleares y de la tecnicalidad proveniente de las fuentes consultadas. Estas se construyen como evidencia desde distintos niveles de calidad, en la elaboración de explicaciones históricas en sus respuestas del género académico "control de lectura de historia” (Henríquez y Canelo, 2014). 
El foco de los estudios en alfabetización histórica ha sido el de caracterizar las formas de razonar, leer y escribir que utiliza la historiografía con el objetivo de transferirlas a los procesos de enseñanza y aprendizaje. En particular, se ha centrado en la caracterización de los procedimientos de lectura de las fuentes empleadas por los historiadores, lo que ha permitido detectar la manera en que las evidencias son analizadas y relacionadas, con el fin de dotarlas de significación histórica. En esta tarea, los procesos de identificación de la perspectiva del autor, la comparación y corroboración de la información con otros documentos (Paxton, 2002; Stahl y Shanahan, 2004) son articulados mediante el uso de estructuras conceptuales en las que se conecta el conocimiento de los hechos y procesos históricos con el contenido retórico de la disciplina. Los conceptos propios de la disciplina entregan los esquemas para organizar la cadena causal de la narración histórica (Paxton, 2002), en tanto los recursos retóricos permiten estructurar discursivamente el conocimiento. Así, la articulación de ambas dimensiones constituye un esquema organizacional que otorga sentido histórico al relato, a través de, por ejemplo, la identificación de los recursos verbales que permiten identificar la jerarquía de las causas, la agentividad humana y la manera en que los historiadores elaboran la significación histórica.

Con este propósito en mente, consideramos que la Lingüística Sistémico Funcional (LSF) podría colaborar a comprender este proceso, dada la perspectiva sociosemiótica de este paradigma, el cual considera que el lenguaje construye y es construido por el contexto social en una acción continua que implica temporalidad ( $\mathrm{Ha}$ Iliday, 1994; Lemke, 1995; Fairclough, 2003), y en el que, por tanto, las relaciones entre el discurso y la experiencia son fundamentales. Halliday (1994) plantea que, como componente intrínseco de todo sistema semiótico, la intertextualidad conlleva las dimensiones temporales de una semogénesis que se expresa como ontogené- sis, filogénesis y logogénesis. De este modo, los significados intertextuales, que son los que se actualizan en la construcción de la evidencialidad en el discurso, implican una negociación de significados ideacionales (de representación de la experiencia) e interpersonales (de interacción de intersubjetividades) en un tiempo determinado y de acuerdo con una jerarquía discursiva que actúa en una matriz semiótica social mayor.

El modelo de valoración propuesto por Martin y White (2005) en el marco de la Lingüística Sistémico Funcional (LSF) se compone de tres sistemas complementarios que dan cuenta de valoraciones positivas y negativas que pueden atribuirse a personas, cosas o fenómenos naturales (sistema de Actitud), las cuales a su vez pueden ser intensificadas o disminuidas, o delimitadas o desdibujadas (sistema de GRADACIÓN) y valoradas desde determinadas fuentes u orígenes (sistema de compromiso). El sistema de actitud se organiza en tres subsistemas: el de afecto, que reúne los recursos utilizados para expresar emociones; el de JUICIO, que comprende los recursos utilizados para juzgar la conducta de las personas en cuanto Estima Social y Sanción Social; y el de APRECIACIón, que organiza los recursos utilizados para atribuir valor a las cosas o fenómenos naturales. No nos detenemos en una explicación más detallada de los dos primeros sistemas, puesto que el último, el sistema de сомpromiso, nos pareció particularmente pertinente para el tratamiento de la evidencialidad, dado que permite reconocer la fuente de los significados interpersonales inscritos o evocados en el discurso. Asimismo, este modelo posibilita la identificación de los recursos lexicogramaticales y su expresión como patrones valorativos en el nivel discursivosemántico utilizados para subjetivizar las voces presentes en el texto. Estos significados pueden articularse, según Martin y White -quienes se inspiran en el trabajo de Bajtín/Voloshinov- en orientaciones monoglósicas y heteroglósicas que los autores escogen para construir respuestas solidarias, contestatarias, desafiantes, com- 
placientes, entre otras, y, de esa manera, no sólo posicionarse como hablantes/escritores en el discurso, sino también en relación con los interlocutores (White, 2003; Martin y White, 2005).

Estas instanciaciones de los diferentes modos de incluir las voces de otros en el discurso pueden a su vez ser exploradas como un espacio topológico de regiones semánticas que influyen gradualmente unas en otras (Fuller, 1995). De tal modo, "el sistema de compromiso, entendido como una manera de expresar la intertextualidad, debe incluir necesariamente la dimensión de evidencialidad si abordamos el análisis de un discurso histórico. Esta evidencialidad puede construirse a través de la elaboración de la información contenida en fuentes primarias, siguiendo una orientación más o menos heteroglósica en su inserción en el discurso" (Oteíza, 2014: 118). La evidencia que los historiadores construyen en su discurso, así como la seleccionada por parte de los estudiantes de las fuentes primarias $y$, en este caso, también de la bibliografía secundaria, es integrada en las respuestas de prueba con una determinada valoración en el discurso. Por razones de espacio, no podemos abordar este aspecto en este trabajo, pero baste mencionar para nuestros objetivos que las inserciones son construidas en la explicación histórica desde una intersubjetividad que se expresa como juicios de estima y sanción social y/o desde determinados afectos y apreciaciones de eventos, procesos y situaciones sociales. Los elementos que se insertan de las fuentes consultadas por los estudiantes se incorporan en sus respuestas de prueba mediante recursos como cláusulas proyectadas mentales y verbales, formas impersonales, pasivas plenas o reflejas, nominalizaciones, polaridad negativa, modalización, modulación, entre otras estructuras lexicogramaticales que permiten por acumulación construir discursivamente la voz de los autores de las lecturas consultadas, y probar — dado que se trata de una instancia de evaluación - que efectivamente han leído y comprendido las fuentes y bibliografías asigna- das para el curso.

Si bien todo enunciado es inherentemente dialógico en tanto siempre es una manifestación de las influencias de "lo que se ha dicho/escrito previamente y que se anticipa a las respuestas de lectores/receptores actuales, potenciales o imaginados" (Martin y White, 2005: 92), la opción monoglósica implica el no reconocimiento o supresión de este dialogismo al ignorarse las otras voces o posiciones. Esta opción monoglósica se instancia prototípicamente a través de enunciados declarativos o afirmativos y uso de estructuras impersonales, cuyos efectos de sentido estarán sujetos a su contexto específico de enunciación. Por otro lado, si los autores/escritores escogen una orientación heteroglósica, están comunicando que, de una u otra manera y en diferentes grados, reconocen la existencia de otras voces y de posturas alternativas. Con el objetivo de dar cuenta de los recursos heteroglósicos, White $(2000,2003)$ propone las nociones de intravocalización y de extravocalización, las cuales dan cuenta de las posibilidades más implícitas o explícitas de multiplicar las voces de un texto. Esta clasificación es complementaria a las de expansión y contracción dialógica (Martin y White, 2005), las cuales dan cuenta respectivamente del grado con el que los enunciados permiten posiciones y voces alternativas o rechazan o restringen el alcance de los mismos.

Dado que el tratamiento de la evidencialidad en el discurso de la historia guarda particular relación con la inclusión de fuentes primarias y bibliografía secundaria en la construcción de explicaciones históricas, los mecanismos de expansión dialógica a través de las categorías de Atribución como Inserción y como Asimilación fueron clave en el análisis de nuestro corpus. Estos mecanismos heteroglósicos se clasifican como de extravocalización. Sin embargo, en numerosas oportunidades los y las estudiantes presentan hechos, condiciones, motivaciones históricas o ideas nucleares de los autores asignados para sus controles de lectura sin mencio- 
narlos explícitamente, incorporando las ideas o planteamientos de la evidencia consultada sin explicitar la fuente de procedencia de lo mencionado. Las ideas de los autores se codifican a través de recursos de contracción dialógica, principalmente de Proclamación como Respaldo, o a través de recursos que colaboran con la construcción de un discurso más monoglósico, pero que "oculta" la evidencia a través de formas impersonales, pasivas, procesos relacionales y materiales en cláusulas afirmativas. Así, desde el punto de vista del tratamiento de la evidencia, los y las estudiantes integran mediante citas indirectas a los autores como Atribución por Asimilación (Guerrero señala...; Viqueira afirma...; el autor sostiene...) o a través de la inserción de la evidencia sin reconocimiento de la misma. En la figura 1 , reproducimos la red sistémica propuesta por Martin y White para el análisis de los recursos lingüísticos que colaboran con los distintos grados de inclusión de las voces de otros en el discurso:

\section{FIGURA 1}

Sistema de compromiso basado en White $(2000,2003)$ y Martin y White (2005)

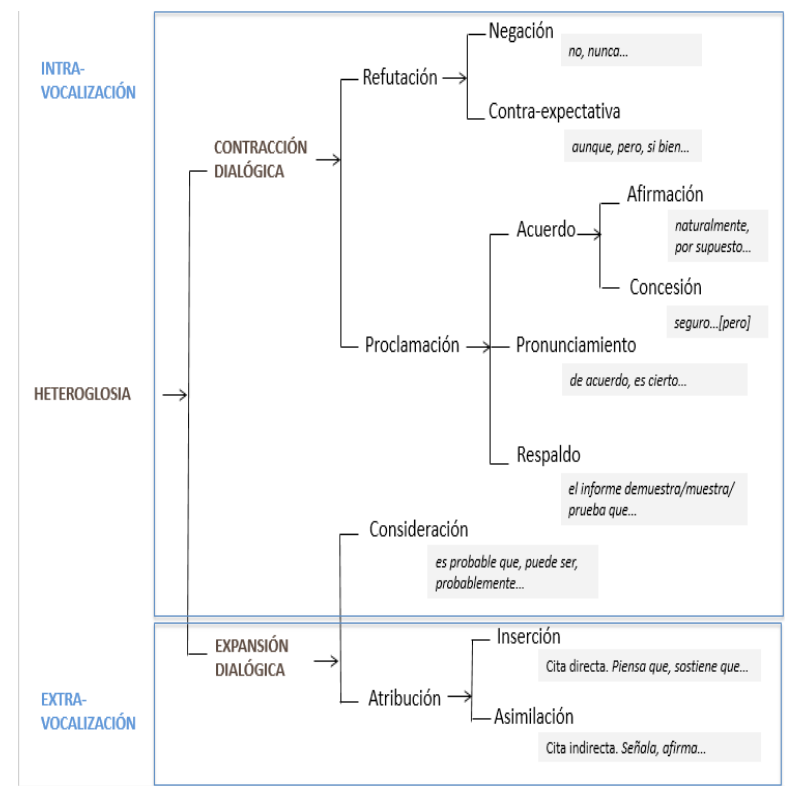

La evidencia histórica, en cuanto elaboración que debe construir el historiador a partir de la información contenida en fuentes primarias o secundarias de diferente naturaleza, constituye una dimensión fundamental de todo discurso histórico. Dado que la historia tiene un carácter interpretativo (Fontana, 1992; Trouillot, 1995; Ricoeur, 2010), es fundamental el trabajo de selección de la información que el/la historiador/a toma de las fuentes primarias para construir una cadena causal como base de una explicación histórica sólida. En el caso particular del corpus considerado en esta investigación, es importante, eso sí, tener en cuenta que las fuentes primarias y bibliografía secundaria son asignadas a los estudiantes; asimismo, en el género histórico académico "prueba de comprensión de lectura", el estudiante debe construir su argumento utilizando estas fuentes de manera obligatoria y no otras, esto es, el estudiante no ha participado en el proceso de selección de fuentes. Lo crítico, entonces, es examinar de qué manera las incluye en su respuesta y la calidad de esta incorporación para la construcción de una explicación histórica convincente.

\section{Análisis y discusión}

En esta sección presentamos el análisis del corpus contemplado para este trabajo. Por razones de espacio nos concentramos en la presentación detallada de dos ejemplos paradigmáticos de respuestas de pruebas de control de lectura para cada nivel. El corpus total de la investigación lo componen 16 respuestas escritas de estudiantes de primer a tercer año de la carrera de Licenciatura en Historia de la Pontificia Universidad Católica de Chile de diferentes cursos de historia de Chile que abarcan desde el siglo XV al siglo XIX, recopilado el año 2012. A continuación, incluimos en el cuadro 1 , a modo de ejemplo, la matriz de análisis de la correlación de la evidencia en la que pueden apreciarse los criterios que tomamos en consideración. Hemos incorporado algunos ejemplos del caso de estudiantes de tercer año para que este cuadro pueda leerse con mayor facilidad: 


\section{CUADRO 1}

Análisis de la correlación de la evidencia

EVIDENCIA: VIQUEIRA, Juan Pedro, 1987: ¿Relajados o reprimidos? Diversiones públicas y vida social en la ciudad de México durante el Siglo de las Luces, Ciudad de México: Fondo de Cultura Económica. (De control de lectura de curso de tercer año Temas de Chile y América Siglos XVI-XVIII).

\begin{tabular}{|c|c|c|c|c|c|}
\hline \multicolumn{2}{|c|}{ SELECCIÓN DE LA EVIDENCIA } & \multirow{2}{*}{\multicolumn{4}{|c|}{$\begin{array}{c}\text { CALIDAD DE LA INTEGRACIÓN² } \\
\text { INDICADORES }\end{array}$}} \\
\hline \multirow[b]{2}{*}{ ELEMENTOS } & \multirow[b]{2}{*}{ EJEMPLOS } & & & & \\
\hline & & $\begin{array}{c}\text { Incorporación } \\
\text { datos no } \\
\text { presentes en } \\
\text { la evidencia }\end{array}$ & $\begin{array}{l}\text { Incorporación } \\
\text { estratégica } \\
\text { y/o errónea }\end{array}$ & $\begin{array}{l}\text { Reformulación } \\
\text { sin juicio }\end{array}$ & $\begin{array}{l}\text { Reformulación } \\
\text { con inferencia, } \\
\text { sentido histó- } \\
\text { rico y/o juicio }\end{array}$ \\
\hline \multirow{2}{*}{$\begin{array}{l}\text { A. Elementos } \\
\text { aislados } \\
\text { seleccionados } \\
\text { (hechos, con- } \\
\text { diciones y/o } \\
\text { motivaciones) }\end{array}$} & $\begin{array}{l}\text { Elite siente la necesidad } \\
\text { de ayudar a la plebe. }\end{array}$ & & & $\begin{array}{c}\text { X } \\
\text { (sólo juicio } \\
\text { de autor) }\end{array}$ & \\
\hline & $\begin{array}{l}\text { Los ilustrados se cansan } \\
\text { de compartir con la plebe. }\end{array}$ & & $\begin{array}{c}\text { X } \\
\text { (incorporación } \\
\text { estratégica) }\end{array}$ & & \\
\hline \multirow[t]{2}{*}{$\begin{array}{l}\text { B. Plantea- } \\
\text { mientos } \\
\text { seleccionados }\end{array}$} & $\begin{array}{l}\text { El pensamiento ilustrado } \\
\text { introdujo en la mentalidad } \\
\text { de las elites nuevas concep- } \\
\text { ciones y valores, con con- } \\
\text { secuencias desde el plano } \\
\text { político hasta cultural (quie- } \\
\text { bre con el mundo conocido). }\end{array}$ & & & & $\begin{array}{c}\text { X } \\
\text { (deducción } \\
\text { del estudiante } \\
\text { a partir de la } \\
\text { evidencia) }\end{array}$ \\
\hline & $\begin{array}{l}\text { Al haberse convertido } \\
\text { en la nueva fiesta tradi- } \\
\text { cional de la sociedad [el } \\
\text { teatro], la elite buscaba } \\
\text { legitimizar su dominación } \\
\text { sobre el pueblo, siguien- } \\
\text { do las ideas ilustradas. }\end{array}$ & & $\begin{array}{c}\mathrm{X} \\
\text { (incorporación } \\
\text { errónea) }\end{array}$ & & \\
\hline $\begin{array}{l}\text { C. Recuperación } \\
\text { de las ideas } \\
\text { nucleares }\end{array}$ & $\begin{array}{l}\text { El factor económico se } \\
\text { contrapuso con los idea- } \\
\text { les ilustrados. Por ello, se } \\
\text { volvieron a los espectáculos } \\
\text { "vulgares", mostrando la } \\
\text { fragilidad del ideario. }\end{array}$ & & $\begin{array}{c}\mathrm{X} \\
\text { (incorporación } \\
\text { estratégica: } \\
\text { simplificación } \\
\text { del proceso) }\end{array}$ & & \\
\hline \multirow[t]{2}{*}{ D. Tecnicalidad } & $\begin{array}{l}\text { Afrancesamiento; llustra- } \\
\text { dos; Reformas Borbónicas; } \\
\text { Despotismo llustrado. }\end{array}$ & & $\begin{array}{c}\mathrm{X} \\
\text { (incorporación } \\
\text { estratégica) }\end{array}$ & & \\
\hline & Segregación social & $\begin{array}{c}\mathrm{X} \\
\text { (concepto } \\
\text { incorporado } \\
\text { por el estudian- } \\
\text { te a modo de } \\
\text { deducción) }\end{array}$ & & & \\
\hline
\end{tabular}




\subsection{Caso 1: Historia de América y Chile S. XV-XVI}

El primer caso que analizaremos corresponde al curso Historia de América y Chile S. XV-XVI, dictado el primer año (primer semestre) de la Licenciatura en Historia. La evaluación seleccionada para el análisis es un control de lectura que incluía tres preguntas de desarrollo, de las cuales escogimos una con el fin de evaluar los patrones de evidencialidad en las respuestas de los estudiantes.

La evidencia considerada en la pregunta corresponde a una fuente primaria denominada "El encuentro con el mundo maya. La relación de Gonzalo Guerrero". Se trata de una crónica del siglo XVI que narra de manera cronológica la historia de Gonzalo Guerrero, un soldado español cuya expedición naufraga en las cercanías de las costas americanas de la península de Yucatán. La fuente relata y describe los hechos y motivaciones que permitieron que Guerrero llegara a la zona del Mayab como esclavo, pero después se convirtiera en el esposo de la hija del jefe local indígena. El núcleo de la crónica lo constituye la narración de la reacción de Guerrero y los acontecimientos desarrollados a partir de la llegada de la expedición de Hernán Cortés a la zona, poniendo en tensión el origen español del protagonista y los lazos afectivos y familiares desarrollados posteriormente con los indígenas locales ${ }^{3}$. El carácter de la evidencia utilizada (fuente primaria, crónica) implica que el relato se construya a partir de la exposición cronológica y continua de hechos, motivaciones y algunas condiciones de los actores involucrados ${ }^{4}$, sin presentarse por ello una tesis o un argumento explicativo que interprete la información entregada. Presentamos a continuación la consigna de la pregunta analizada y luego dos ejemplos de casos representativos de respuestas de estudiantes.

\section{CONSIGNA}

La relación de Gonzalo Guerrero encarna una fuente clave para el entendimiento del conflicto entre españoles e indígenas en la zona maya del Yucatán. Caracterice dichas particularidades desde la activa dinámica desarrollada entre las expediciones realizadas por los españoles hacia la zona y, a su vez, la concepción y actitud del indígena respecto de este conquistador europeo. Utilice la figura de Guerrero como un actor principal y a la vez único dentro de tal situación.

2 Las definiciones de los indicadores de calidad fueron elaboradas a partir del análisis de las respuestas de los estudiantes y las presentamos a continuación:

- Incorporación estratégica y/o errónea: El estudiante retoma elementos de la pregunta y/o conceptos abordados por el autor sin explicarlos o reformula sus ideas de forma incompleta. No hay una elaboración de los elementos tratados en la evidencia; por lo tanto, la exposición es simple y carece de abstracción.

- Reformulación sin juicio: Se mantienen las ideas centrales de la evidencia, sin la incorporación del punto de vista del estudiante.

- Reformulación con inferencia, sentido histórico y/o juicio: El estudiante incorpora planteamientos de la evidencia y los integra en su escritura a través de un proceso deductivo que le permite construir sentido histórico.

3 Algunos de los hechos que menciona el cronista son episodios de la historia personal del protagonista, como su llegada a tierra firme, la captura de Guerrero por los indígenas, el matrimonio entre Gonzalo y la princesa y la celebración del nacimiento del hijo mestizo. También se abordan hitos vinculados con la historia global de la conquista de México, como la llegada de nuevas naos con españoles y caballos, la derrota de los españoles, la congoja de Guerrero, el regreso de los españoles, la organización de los indígenas para la batalla, la superioridad militar española, la llegada de los españoles y solicitud de enrolarse a Guerrero (negativa de Guerrero), el Ilamado de Cortés a cooperar, las noticias de la caída de Tenochtitlán, la administración opresiva de Cortés y sometimiento (religioso y social) de los indígenas, entre otros.

4 Algunas motivaciones mencionadas por el cronista se relacionan con las emociones y sentimientos de los personajes: Ia hija del rey gustó de Guerrero, pena por la posible destrucción del pueblo, agrado de Guerrero por la vida que llevaba entre los mayas (vergüenza por no unirse a las armas), pena y rabia en Mayab por la crueldad española, orgullo de los españoles que no se devolverán a España, negativa de los indígenas a la conversión religiosa. Algunas condiciones mencionadas por el cronista: descubrimiento por Colón que permitió sumar nuevas tierras a Castilla, mayor tecnología militar española; superioridad numérica indígena, superioridad militar española, sometimiento de Tenochtitlán; administración opresiva de Cortés, expansión de la conquista militar española. 


\section{Ejemplo 15:}

Después del naufragio, Guerrero vio perdida toda opción de sobrevivencia, sobre todo al ver que ninguno de sus compañeros había sobrevivido. Sin embargo, Guerrero es adoptado por esta nueva cultura e integrado como un miembro más. Al ofrecerle comida junto a ellos, al vestirlo con sus propias túnicas, el pueblo maya muestra su disposición a aceptarlo.

Quizás, puede entenderse como una estrategia en un principio para poder entender al adversario. Pero, con el tiempo, esta nueva cultura puede ver en él atributos que ellos también valoraban. Él es capaz de elaborar un barquillo, que representa la capacidad para utilizar sus manos como un artesano. Este aspecto es sin duda un nexo entre ambas culturas, que le permite acceder a ella, a su idioma y establecer vínculos familiares entre ellos.

En relación a los primeros contactos la aculturación española era evidente, la religión era necesariamente la única opción de creencias. Para el nativo, sin duda este era el aspecto más importante y es por esta razón, el pueblo Maya se ofende cuando llegan misioneros a intentar agraciarlos con la imposición de su cultura y religión (...).

El sincretismo, base del logro de España, se manifiesta en la no pérdida de la identidad y el reflejo de la unión de concepciones y creencias imposibles de desarraigar. Tanto para Guerrero como para los nativos la importancia de la religiosidad es intransable (ID 17).

El relato anterior se estructura principalmente a partir de lo que hacen, piensan y sienten los actores sociales, centrando la argumentación en la actitud de los indígenas hacia Gonzalo Guerrero y su disposición a aceptarlo. Para ello se mencionan algunos hechos y se evalúa la actitud de los actores involucrados en dicha situación a través de valoraciones de Afecto (el pueblo maya se ofende) y juıcıo de estima y sanción so- cial (Guerrero es adoptado por esta nueva cultura e integrado; el pueblo maya muestra su disposición a aceptarlo). De esta manera, el relato es construido en un menor nivel de abstracción, a pesar de que el o la estudiante incluye elementos técnicos que no están presentes en la crónica (aculturación, pueblo maya, sincretismo).

En cuanto a la construcción de la evidencialidad, en este primer ejemplo predomina la utilización de heteroglosia por intravocalización de contracción dialógica, a través de marcas de Proclamación de Pronunciamiento: sin duda (dos veces) y era evidente, y recursos de Refutación de Contraexpectativa (pero, sin embargo). Mediante el análisis podemos dar cuenta de que se trata de un recurso estratégico, en la medida en que dichas expresiones establecen como necesarias y evidentes las consecuencias de los hechos/conceptos presentados en la respuesta (sin alternativas), para darle claves al lector/corrector sobre la lectura y comprensión de la crónica, aunque las relaciones establecidas no se encuentran en la fuente primaria.

Asimismo, en el relato no se alude a las condiciones mencionadas por el autor en la crónica, que le permitirían al estudiante caracterizar de mejor forma el conflicto entre españoles e indígenas en el cual se centra la consigna. La respuesta del estudiante simplifica las motivaciones de los actores sociales, privilegiando la exposición cronológica de algunos de los hechos rescatados de la crónica, los cuales son utilizados como las "garantías evidenciales" de la respuesta. Estos hechos brindan señales al evaluador de que el estudiante leyó la crónica para el control de lectura, aunque es interesante destacar que sólo se seleccionan algunos de los múltiples hechos presentados en la fuente primaria (ver nota a pie de página 3). La calidad de la selección se sitúa

5 Los elementos señalados en los ejemplos muestran recursos para construir el sistema de compromiso en el discurso. Los recursos de orientación más heteroglósica están subrayados con letra normal (procesos mentales y verbales, modalización, modulación, polaridad negativa, concesión, entre otros) y los de orientación más monoglósica subrayados con cursiva (procesos relacionales o materiales en cláusulas afirmativas, formas impersonales y pasivas, nominalizaciones, entre otros). Estos recursos de diferente naturaleza son considerados para el análisis de la evidencialidad. 
mayoritariamente en la incorporación estratégica o errónea de los hechos y motivaciones, y en la reformulación sin juicio (Guerrero vio perdida toda opción de sobrevivencia; el pueblo maya se ofende cuando llegan misioneros). El estudiante no presenta incorporaciones que incluyan inferencias con sentido histórico y/o juicios propios, que señalen perspectiva del estudiante sobre la evidencia. Se trata, por lo tanto, de una simplificación de los hechos presentados en la crónica, mencionados sintéticamente en la respuesta.

A raíz del carácter de la crónica no hay otros planteamientos seleccionados ni otras ideas nucleares del autor que puedan ser retomadas por el estudiante. Cabe destacar, sin embargo, que en este ejemplo el o la estudiante utiliza conceptos técnicos que permiten teorizar sobre la crónica (aculturación, pueblo maya, sincretismo) que no están presentes en la evidencia.

\section{Ejemplo 2:}

América es un continente sumamente diverso, por lo que la concepción y rectitud del indígena respecto al conquistador europeo no siempre fue la misma. La zona Maya del Yucatán fue de los primeros del continente en tener contacto directo con los españoles los colonizadores llegaban en sus Naos y caballos a estas tierras. Estos elementos eran desconocidos por los indígenas, tanto como otros como por ejemplo el acero. Por lo demás, la apariencia física del europeo era totalmente distinta. Fuentes españolas nos dicen que en el caso de los Aztecas es probable que éstos hayan intentado explicar la llegada de Cortés relacionándolo con su mitología, y que por eso lo reciben tan hospitalariamente. Sin embargo, esto no está comprobado, puesto que también se plantea que Moctezuma puede haberlo hecho entrar para tenerlo cerca y que era costumbre hacerlo, por lo demás casi podría matarlo más fácilmente si lo veía como una amenaza.

En el caso de Yucatán se da la particularidad de que los indios primero que todo reciben a Gonzalo Guerrero seguía sus propias tradiciones como lo hacían con los extranjeros, lo esclavizan. Sin embargo se da la particularidad de que el pri- sionero es tratado de manera bastante hospitalaria, e incluso se gana la amistad de los jefes al demostrar su talento en la carpintería, que luego enseña al hijo del jefe junto al arte de la esgrima.

De esta manera se evidencia que los indígenas no estaban cerrados a un contacto cultural en el que pudieron incorporar elementos europeos como propios, aunque en este caso también sea con motivos de aprender a defenderse del español.

Además, se muestra como eran capaces de incorporar a estos extranjeros a sus pueblos, como lo demuestra el matrimonio de Guerrero con la Hija del jefe, la más agraciada del pueblo.

Por otro lado, también era claro como esta relación, sin embargo, no era de sumisión al español. Los mayas de Yucatán se sentían amenazados por las grandes Naos y los desconocidos caballos, y peor aún, se sentían agraviados al darse cuenta que los españoles venían a imponer una religión y costumbres que no eran propios de ellos, de lo que en el caso de Guerrero se enteraban una vez que el español se los dijo (antes del contacto directo con los colonizadores) pero en otros casos no se enteraban hasta que los españoles se asentaron e intentaron evangelizarlos y asimilarlos a la cultura europea. En ese sentido, muchos indígenas (como por ejemplo los tlaxcaltecas) fueron engañados (ID 21).

Al igual que en el primer ejemplo, en este caso el relato se estructura fuertemente a partir de lo que hacen y piensan los actores sociales involucrados en el discurso: indígenas, aztecas, Moctezuma, Gonzalo Guerrero, los españoles. La argumentación se construye principalmente a través de la exposición de la capacidad y la disposición de los actores involucrados en el conflicto, utilizando valoraciones de juıcıo de estima y sanción social (Además, se muestra cómo eran capaces de incorporar a estos extranjeros a sus pueblos; lo reciben tan hospitalariamente).

Con respecto a la construcción de la evidencialidad, en el segundo ejemplo se aprecia un uso equivalente tanto de heteroglosia como de monoglosia. La heteroglosia está dada por la in- 
corporación de las voces de los actores sociales en el discurso, así como mediante el recurso de contracción dialógica, de Refutación por Contraexpectativa (sin embargo, aunque, pero) y de Refutación por Negación (no siempre fue, no está comprobado, no estaban cerrados, entre otros). Por otra parte, la monoglosia se expresa en cláusulas afirmativas con procesos materiales y relacionales y procesos impersonales, en donde los hechos se presentan como necesarios y evidentes, sin que el estudiante construya una explicación acabada sobre por qué ocurren. Lo anterior se ilustra especialmente en la utilización de estructuras impersonales (se da, se gana, se evidencia) y pasivas (estos elementos eran desconocidos). En ese sentido, a diferencia del primer ejemplo, la ocurrencia "necesaria" de los hechos se construye a través de las cláusulas afirmativas impersonales y también a través de recursos que indican heteroglosia por intravocalización

Asimismo, en este segundo ejemplo el o la estudiante utiliza recursos de intravocalización de Proclamación por Respaldo con uso de verbos de evidencialidad impersonales para referirse a la información de la crónica: se plantea, se muestra, se evidencia, sin reformular la evidencia o simplificando la sucesión de los hechos seleccionados. Por último, cabe destacar que se mencionan fuentes españolas a pesar de que para esta pregunta se considera sólo la crónica de Gonzalo Guerrero. Se pondera parcialmente la información presentada (actitud de los aztecas con los españoles) a través de recursos que marcan heteroglosia de intravocalización de expansión dialógica de Consideración (probable) o de extravocalización de Atribución por Asimilación (nos dicen) y mediante recursos de intravocalización de Refutación de Contraexpectativa (sin embargo esto no está comprobado), si bien se trata de perspectivas que no están presentes en la fuente primaria. A diferencia del primer ejemplo analizado para este nivel, la utilización de informa- ción ausente en la crónica no responde al eje de la tecnicalidad, sino a planteamientos generales del tipo América es un continente diverso.

\subsection{Caso 2: Historia de América y Chile S. XIX}

El segundo caso que caracterizaremos corresponde al corpus obtenido del curso Historia de América y Chile S. XIX, que se dicta el segundo año (primer semestre) de la Licenciatura en Historia. La evaluación seleccionada es un control de lectura que incluye tres preguntas de desarrollo, una de carácter obligatorio y las otras dos optativas, por lo tanto, los estudiantes podían escoger una de estas últimas. Para la realización de nuestro análisis, consideramos la pregunta obligatoria con el fin de evaluar los patrones de evidencialidad en las respuestas de los estudiantes. A continuación presentaremos dos ejemplos paradigmáticos de los seis casos analizados para este curso.

Para contestar la pregunta, los estudiantes debían incorporar dos evidencias correspondientes a bibliografía secundaria. Por una parte, el texto de François-Xavier Guerra, "Modernidad e Independencias. Ensayos sobre las revoluciones hispánicas" (1992), que aborda dos conceptos claves para comprender el proceso de autonomía de las colonias americanas con respecto a la metrópoli: la modernidad (mutaciones culturales, del imaginario, comportamientos y valores, asociados también a la Revolución francesa y a la llustración) y la independencia política (procesos de independencia americana luego de la invasión napoleónica a la península ibérica y las abdicaciones de Bayona en 1808). La tesis principal apunta justamente a diferenciar ambos procesos, que, a pesar de que se producen en un espacio temporal similar y están muy relacionados, no pueden confundirse para comprender a cabalidad el desarrollo de las independencias americanas ${ }^{6}$. Para el autor, la relación entre ambos fue distinta considerando los casos de la

6 La tesis en palabras del autor: "Hay pues, que separar dos fenómenos que se producen al mismo tiempo y están muy 
metrópolis y de América: mientras en la primera la modernidad fue uno de los antecedentes para el desarrollo de la revolución política, en la segunda la Independencia de la península no necesariamente fue precedida por una mutación cultural y una revolución (cambios sociales y políticos nuevos, que rompen completamente con el pasado)7.

Por otro lado, los estudiantes debían considerar el texto de Manuel Chust Calero "La coyuntura de la crisis: España, América” (2003), que corresponde a bibliografía secundaria de tipo monográfica, estructurada a partir de un argumento central y la exposición de una serie de hechos, motivaciones y condiciones referidos al desarrollo del proceso de revolución política e independencia hispanoamericana. La tesis central del autor postula que la invasión napoleónica a la península ibérica y las abdicaciones de Bayona de 1808 "van a precipitar la crisis de la monarquía absoluta española. Empieza la denominada, para la península, Guerra de la Independencia que, paradójicamente, también provocará el inicio de las guerras de independencia americanas" (Chust Calero, 2003: 55). En comparación con el texto de François-Xavier Guerra, este libro se centra mucho más en la exposición de hechos (tanto antecedentes como consecuentes) que son complementados con motivaciones y condiciones para explicar los procesos de independencia americanos ${ }^{8}$. Presentamos a continuación la consigna de las respuestas analizadas que considera las dos evidencias anteriores:

\section{CONSIGNA}

Explique por qué François-Xavier Guerra diferencia entre Revolución política e irrupción de la Modernidad. Con el texto de Chust Calero, desarrolle en forma general ambos procesos y haga énfasis en la actuación de las Cortes de Cádiz y el debate suscitado al respecto en América.

\section{Ejemplo 3:}

François-Xavier Guerra, en su libro «Modernidad e Independencia» trata el tema de cómo en América se van suscitando los movimientos independentistas, teniendo como base del análisis los conflictos políticos que se suscitan principalmente entre 1808 y 1809 y además toma el tema de la Modernidad como nuevo sistema de pensamiento que va modelando las conciencias de los criollos, el cual le ayuda a aprovechar el momento político que se está dando en el contexto.

Aunque Guerra tiene esa base, no dice que ambos procesos son idénticos o van provocando los mismos resultados, tanto en América como en la península, sino que se hace una referencia a que si bien trabajan dentro del mismo contexto

6 imbricados, pero que no pueden confundirse como lo muestra muy claramente el caso de Brasil: la independencia de las metrópolis y la revolución, es decir, la adopción brusca de un sistema de nuevas referencias políticas y sociales que intentan hacer tabula rasa del pasado" (Guerra, 1992: 19-20).

7 Algunas de las condiciones culturales consideradas por el autor: ilustración ibérica (nueva visión del hombre y de la sociedad), difusión de nuevas formas de sociabilidad, libertad de prensa; condiciones políticas y sociales: relaciones entre Estado y sociedad, absolutismo, inexistencia de instituciones representativas. Algunas motivaciones: descontento de la elite con absolutismo, sentimiento de necesidad de representación de la sociedad ante el Estado, sentimiento americano de inferioridad frente a los españoles peninsulares, rechazo hacia el invasor y fidelidad a Fernando VII. Algunos hechos: invasión de Napoleón a España en 1808, constitución de la Junta Central administrativa en septiembre de 1808, elecciones americanas para enviar diputados a la Junta central en 1809, elaboración de una nueva Constitución en 1812, formación de las Juntas Americanas.

8 Algunos de los hechos expuestos por Chust Calero: dominio militar de ejércitos napoleónicos en 1807, Abdicaciones de Bayona, rebelión popular española en mayo de 1808, formación de Junta Central en 1808, convocatoria a Cortes, extensión de Juntas a territorios americanos, proclamación de Constitución 1812, Congreso de Viena, formación de la Santa Alianza, restitución de Fernando VII como rey absoluto, muerte de Fernando VII, entre otros. Algunas motivaciones: interés de Napoleón de incorporar territorios españoles, preocupación de Fernando VII por caída del absolutismo y pérdida de territorios americanos, esperanza de los americanos por representatividad ante la nueva junta y su posterior descontento con representación insuficiente. Algunas condiciones (políticas, sociales y culturales): Reforma Ilustrada, Revolución Liberal, circulación de la información entre España y América, contexto internacional favorable hacia monarquías, crisis de la hacienda monárquica española. 
temporal, tienen particularidades bien marcadas que les dan su propia identidad. Guerra se preocupa más sobre el asunto de la identidad individual, la modernidad. Para poder explicar de mejor medida la Revolución Política, se tomará a Chust Calero, quien explica de mejor medida $\underline{10}$ suscitado entre la abdicación de Fernando VII en Bayona y las Cortes de Cádiz.

La irrupción de la modernidad es un proceso ideológico que va transformando la visión que tienen, tanto españoles como americanos, de sí mismos; esto quiere decir que el punto principal de la modernidad, en una primera instancia, tiene que ver con la transformación del concepto de nación. (...)

Esto es la irrupción de la modernidad, pero existe además la revolución política. Esta revolución política tiene momentos muy marcados y la mayoría se dan en el concierto europeo, va desde la abdicación de Fernando VII en Bayona, a manos de Napoleón Bonaparte. Cuando el Rey es tomado preso, Napoleón pone a su hermano, José al trono, lo que genera un descontento generalizado en el pueblo, haciendo que estos tengan el derecho de organizarse por sí mismos, lo que genera la Junta Central, esta junta estaba encargada de mantener el poder resguardado para el monarca hasta su regreso, obviamente esto tomó parte en América, la cual por el proceso de la modernidad, se sentía tan ciudadano como el peninsular. Cuando se le piden los Diputados a América, al llegar, la estructura de orden había cambiado y estaba la regencia, este era el poder central que trataba de ordenar a todo el territorio, pero nuevamente no se le tomó en cuenta los americanos en la práctica (...) En muy resumidas cuentas, la revolución política lo que hizo fue darle el empujón, fue abrirle los ojos a América para que ésta comenzara su proceso.

En conclusión, Guerra diferencia a ambos procesos ya que en cada uno de ellos se van generando instancias propias de poder concernir a América como tal y de crear un sentimiento americano, general y luego particular, que sumado a la concepción de modernidad, nación, individuo, etc, forman la independencia del territorio. Deja entender que estos procesos no funcionan por separado, sino que la suma de ambos crean el proceso completo en sí (ID 25).

El relato del o de la estudiante constituye una narrativa estructurada principalmente a partir de la exposición cronológica de las condiciones culturales y algunas condiciones políticas, que le permiten definir parcialmente el concepto de modernidad tratado por François Xavier Guerra. Por tanto, el relato se construye a través de la sucesión de procesos como modernidad (cambio de ideas del individuo, cambio en el concepto de nación) y revolución política, valorados a través del sistema de APRECIACIón ( La irrupción de la modernidad es un proceso ideológico; La revolución política lo que hizo fue darle un empujón, fue abrirle los ojos a América), y presentando en un segundo plano las motivaciones de los actores sociales involucrados. Asimismo, las condiciones se exponen de manera simplificada, resumiendo ampliamente las propuestas de ambos autores y sin presentar explícitamente sus tesis o argumentos principales.

En relación a la construcción de evidencialidad, en este ejemplo destacamos la utilización de heteroglosia de extravocalización de Atribución por Asimilación, utilizada por el o la estudiante para referirse explícitamente a las propuestas de los autores a través de marcas textuales como François-Xavier Guerra, en su libro "Modernidad e Independencia" (toma, le ayuda); Aunque Guerra tiene esa base, no dice; Guerra se preocupa; Se tomará a Chust Calero, quien explica de mejor medida; Guerra diferencia; Deja entender. Si bien este tipo de recursos de extravocalización hacen explícita la referencia a ambos autores, es interesante notar que en la mayoría de las menciones se simplifican ampliamente las propuestas de estos, o bien no se introducen claramente sus argumentos centrales. Por ejemplo, el estudiante plantea que Para poder explicar de mejor medida la Revolución Política, se tomaráa Chust Calero, quien explica de mejor medida lo suscitado entre la abdicación de Fernando VII en Bayona y las Cortes de Cádiz, sin explicar por qué 
dicho autor ofrece una mejor explicación ni profundizar esta perspectiva.

Por lo anterior, puede argumentarse que en algunos casos la mención a los autores constituye un recurso estratégico para "dar guiños" al lector/corrector sobre la lectura de ambos textos, sin que se introduzcan las propuestas nucleares claramente. Por lo mismo, destacamos que la utilización de este tipo de recursos de extravocalización de Atribución por Asimilación se realiza especialmente al inicio y al final de la respuesta, a modo de introducción y conclusión. En el cuerpo y núcleo central de la narración del estudiante se mencionan algunos de los hechos, y condiciones (muy pocas motivaciones), sin explicitar las voces de los autores, utilizando recursos de heteroglosia por intravocalización de contracción dialógica de Proclamación por Acuerdo (obviamente) y recursos monoglósicos a través de cláusulas afirmativas con procesos relacionales atributivos (es, tiene) y procesos materiales y existenciales impersonales (se dan, se está dando, se suscitan, entre otras). Asimismo, el o la estudiante organiza internamente el argumento mediante estructuras textuales como en muy resumidas cuentas, en conclusión, esto quiere decir.

Ejemplo 4:

El autor Guerra hace énfasis en dos fenómenos que hay que distinguir por separado, la revolución política y la mutación cultural.

Guerra entiende por Revolución política a la independencia y a todos los acontecimientos que la rodean, lo cual será especificado más adelante con Chust Calero, y mutación cultural engloba el proceso de modernidad con sus características.

El autor cuestiona si ambos conceptos se encuentran unidos o separados: ¿Revolución política implica una mutación cultural? Según Guerra no, en América por ejemplo la Revolución Política se presentó de manera mucho más rápida que la mutación cultural la cual fue evolucionando de manera paulatina y lenta, debido a la mentalidad tradicional y más cercana al antiguo régimen que a las nuevas ideas que despertaban. En Europa, la mutación cultural si fue acompañada por la Revolución Política, debido a que esta se inicio por las masas "de abajo" en cambio en América fue la elite, la aristocracia quien llevó ese proceso y quien poseía mayor cercanía a la monarquía y a sus principios. La Revolución Política en América tiene su punto clave en 1808 con las Abdicaciones de Bayona (información obtenida gracias al autor Chust Calero), es un hecho importante ya que con el traspaso de la corona de Carlos IV a José Bonaparte, hermano de Napoleón, un grupo de españoles xenófobos se rebela, Esta revolución social recae en la creación de una Junta Central. Para América este hecho es importante ya que en el Decreto de la Junta se declara a América como reino, no más como colonia. La importancia de que sea considerada un reino es que se ve como parte de la Monarquía Española, por lo tanto posee los mismos deberes y derechos y, más relevante aun, misma posibilidad de representación, por lo tanto son llamados a la Junta Central cuatro diputados correspondientes a los Virreinatos de América. Es necesario realizar un paréntesis y recalcar que el Decreto que ve a América como reino, empieza a ser asunto de cuestionamiento para los americanos, los cuales se ven a si mismos como, antes colonia y aquello no les empieza a gustar. (...)

Calero expone el mayor logro de las Cortes de Cádiz, el cual fue la constitución de 1812. Es importante primeramente por que fue jurada y practicada en América y en la península, el autor señala también que sirvió como guía a las futuras constituciones de América (...)

Esta serie de cambios y acontecimientos van erosionando cada vez más la relación entre América y Península, lo cual va a estar acompañado de lentas mutaciones (en el caso de América) culturales que van a desencadenar en un cambio profundo e irreversible (ID 27).

La respuesta anterior se articula a partir de los hechos que aparecen mencionados tanto en el texto de François Xavier Guerra como en el de Manuel Chust Calero. En este sentido, el o la estudiante identifica correctamente algunos de los hechos centrales (abdicaciones de bayo- 
na, junta central, constitución de 1812) que le permiten estructurar la respuesta a partir de los efectos que tuvieron principalmente en el territorio americano. Se mencionan numerosas motivaciones de los actores sociales americanos: son su capacidad, su descontento y su insatisfacción los que hacen de motor de los hechos en esta respuesta, siendo valorados intercaladamente a través del sistema de apreciación (en América por ejemplo la Revolución Política se presentó de manera mucho más rápida que la mutación cultural) y el sistema de juicio de estima y sanción social (en América fue la elite, la aristocracia quien llevó ese proceso y quien poseía mayor cercanía a la monarquía y a sus principios; Un grupo de españoles xenófobos se rebela).

Desde esa perspectiva, en cuanto a la construcción de la evidencialidad, en este ejemplo también predomina la utilización de heteroglosia de extravocalización de Atribución por Asimilación, a través de marcas textuales como: El autor Guerra; Guerra entiende; será especificado más adelante con Chust Calero; el autor cuestiona; según Guerra; información obtenida gracias al autor Chust Calero; Calero expone; el autor señala también.

A pesar de que en ambos ejemplos presentados predomina la utilización de heteroglosia por extravocalización de Atribución por Asimilación, es en este caso donde más claramente la evidencia constituye un aspecto fundamental en la respuesta. Esto se aprecia en que existe mayor cantidad de instanciaciones que introducen explícitamente las voces de los autores, pero también en que dichas introducciones están acompañadas de los argumentos de Guerra y Chust Calero (no sólo es estrategia), donde el estudiante explicita las informaciones obtenidas de cada uno de los textos, a pesar de que en algunos casos simplifica los procesos.

Finalmente, en ambos casos la consigna presentada da el contexto e influencia la manera en que los estudiantes construyen la evidencialidad. Así, al preguntar por dos textos y la identificación de sus argumentos centrales, los estudiantes utilizan recursos heteroglósicos de extravocalización de Atribución por Asimilación para incorporar las voces autorales, a pesar de que la calidad de dicha integración no implique una reformulación de sus planteamientos o constituya una estrategia.

\subsection{Caso 3: Temas de Chile y América Siglos XVI-XVIII}

El tercer y último caso corresponde al corpus obtenido del curso Temas de Chile y América Siglos XVI-XVIII, el cual se dicta en el tercer año (primer semestre) de la carrera de Licenciatura en Historia. En este caso, seleccionamos una evaluación de carácter similar a los dos casos anteriores, es decir, un control de lectura que incluía dos preguntas de desarrollo, de las cuales escogimos una para nuestro objetivo de analizar los patrones de evidencialidad que logran construir los estudiantes en sus respuestas. Presentaremos dos de los seis casos estudiados en profundidad.

Para esta prueba los estudiantes debían leer un libro considerado como bibliografía secundaria, titulado "¿Relajados o reprimidos? Diversiones públicas y vida social en la ciudad de México durante el Siglo de las Luces" de Juan Pedro Viqueira Albán (1987). En este libro el autor analiza el caso de México en el siglo XVIII a través del supuesto "relajamiento" (que se produce en la sociedad popular tradicionalmente conceptualizado de esa manera). Viqueira postula que la elite y las autoridades mexicanas experimentan un cambio de mentalidad influenciadas por las ideas ilustradas y de la modernización, las cuales fueron acompañadas por una intolerancia en general hacia la vida de las clases populares y, de manera particular, a sus diversiones. Esto motivó a la elite y a las autoridades a considerar la necesidad de traspasar las ideas ilustradas a las clases populares y a reformar sus diversiones y, entre ellas, el teatro, dado que era una de las pocas actividades difundidas e incentivadas por la elite. Debido a que el arte dramático era un medio 
propicio para educar a la población, en su gran mayoría analfabeta, se decidió transformarlo e implantar el realismo como única forma permitida para crear una obra y modelar el comportamiento e ideas que el pueblo debía aprender. Viqueira afirma que las reformas no son respuesta a una situación local de relajamiento, sino que más bien es un eco de la nueva intolerancia de la elite y la mentalidad burguesa que surgía en Europa entre las clases dominantes ${ }^{9}$. En esta bibliografía secundaria el autor desarrolla un argumento y una tesis/pregunta central sobre la situación de la sociedad mexicana y enfatiza en su explicación histórica las problemáticas sociales, políticas, económicas y culturales utilizando hechos consecuentes para ejemplificar las premisas centrales ${ }^{10}$

Así, para este control de lectura la consigna o propósito elaborado por el profesor fue el siguiente:

\section{CONSIGNA}

Según lo planteado por Viqueira, ¿Cómo se manifiesta la transformación de valores y principios experimentada en el siglo de las luces por la elite novohispana en la reforma llevada a cabo en el teatro? ¿De qué manera el factor económico pugna con la implementación de estos nuevos ideales en el espacio referido?

\section{Ejemplo 5:}

A lo largo de la colonia veremos cómo la clase dominante comienza a afrancesarse con la incorporación de los valores de la ilustración por lo que sus paradigmas comienzan a cambiar, como también cambia la visión que tienen del otro. Es así como bajo el ejemplo del teatro veremos estas transformaciones y cómo su ideal comienza a verse truncado por circunstancias económicas.

En un primer momento Viqueira nos plantea la nueva concepción que el mismo hombre tenía consigo mismo (...). Asimismo, la élite se sentía con la responsabilidad de ayudar a la plebe normando su comportamiento en su vida cotidiana, con el mejor ejemplo de implementar en el teatro, actividad que se venía realizando hace mucho tiempo representando los valores de la iglesia, ahora lo hicieron representando los valores ilustrados. Tenemos que tener en cuenta que la mayoría de la población era analfabeta, por lo tanto el teatro se convertía así en una de las mejores maneras para educar a la población.

Es de esta forma en donde la élite ve en el teatro la mejor herramienta, en donde además en él confluían todas las clases sociales. La élite durante estos espectáculos veía que la parte de la plebe tenía un comportamiento relajado, al hacer gran desorden en cada uno de los espectáculos, como también con la distracción de los hombres durante la misma obra. Esto se daba ya que las obras más cultas o referidas a temas distintos de la comedia no interesaban a la plebe. Hacían reformas hacia el contenido sin pregun-

9 Algunas de las condiciones económicas sociales y culturales que menciona el autor: el siglo XVIII como época de grandes cambios económicos, sociales y culturales en la Nueva España (afrancesamiento de las costumbres de la elite novohispana, penetración del pensamiento ilustrado, aumento de la población, reformas borbónicas como la reorganización de las haciendas, creación de intendencias, entre otras; decadencia de España manifestada en enfrentamientos políticos, corrupción, disminución de envíos de metales preciosos desde las colonias, entre otros; proceso de mestizaje disminuye las fronteras sociales). En relación al teatro, las condiciones expuestas por el autor son: el teatro como diversión cultural arraigado en las clases bajas; integración del teatro a la vida urbana de Nueva España. Hechos: (antecedente y motivación) Motín de 1692 de indios y castas contra el gobierno español. Autoridades españolas temieron que un posible relajamiento de las costumbres del pueblo desencadenara más motines. Utilización del teatro por parte de los gobiernos virreinales para legitimar el poder desde 1880.

10 Premisa central de Viqueira planteada como tesis/pregunta. "La multiplicación de las leyes y decretos para poner fin a ciertas diversiones públicas sin duda indica que algo había cambiado en la Nueva España. ¿Pero aquello que había cambiado era realmente la sociedad? ¿Será verdad que ésta se había relajado? ¿no será más bien el Estado el que había dejado de ser el mismo y que por tanto no podía seguir tolerando prácticas sociales que eran usuales desde hacía ya bastante tiempo? O bien, ¿serán los dos, el Estado español y sociedad novohispana, los que se habrían modificado profundamente en este siglo? En realidad, sabemos con plena certeza que el Estado español y con él los estratos más altos de la sociedad novohispana, sufrieron una radical transformación y entraron con paso firme a la modernidad, pero lo que desconocemos es si las clases populares siguieron a su manera el camino del cambio en ese siglo" (Viqueira, 1987: 19). 
tarles como lo hacía el despotismo ilustrado que atravesaba la época (...).

Además se ve cómo tanto ilustrados como el Estado no les convenía que la plebe se fuera del coliseo, ya que estos alaban el sustento económico tanto para mantener el teatro como a los actores. Es así como la élite de cierta manera deja de lado todo lo que se querían proyectar con la ilustración para dar un beneficio económico a estas propias obras, comenzando a proyectar comedias y dejando que la plebe se relajara en su actuar.

Bajo todos estos cambios se culmina con el deterioro de lo que en un principio veía en el teatro, ya no educaba a la plebe, sino que esta se había tomado el poder. Cuando los ilustrados se cansan de compartir con la plebe y sus costumbres es cuando deciden separar el teatro para los que de cierta forma valoraran el fin de rescatar valores ilustrados, permitiendo acomodar sus conductas con las que estaban dando en Europa, con reglas más claras con las reformas borbónicas impuestas, a la vez y los que simplemente veían el teatro como una diversión, pero sin esperar que este les entregaran los valores que en ese momento estaban preocupando a la élite.

Es de esta manera como vemos que la élite ejerce un control de la vida de la plebe, por lo que sin ellos cambian sus patrones de comportamiento toda la sociedad los debe seguir. Es así como Viqueira nos habla de relajados y reprimidos, ya que la plebe se comportaba como siempre lo había hecho pero ahora los reprimen con fuertes normas para que cambiaran su comportamiento y así se parecieran a lo que la élite tenía proyectado como el estereotipo ideal de comportamiento, con el ideal de la (...), que a la vez buscaban en otros comportamientos de la vida cotidiana, pero que muchas veces dejaban de lado para que el Estado no dejara de recibir los recursos necesarios (ID 33).

El o la estudiante de este ejemplo construye su relato como una narrativa que se expresa en parte por la presencia de actores humanos y por la imprecisión temporal en la secuencia de eventos en el que predomina el uso de tiempo presente y pasado con un uso cronológico. El discurso se personaliza desde las emociones y sentimientos de los participantes (no interesaban a la plebe; los ilustrados se cansan) y no desde procesos sociales, culturales, políticos u otros, produciendo una menor abstracción en la construcción discursiva. Así, el relato es construido a partir de los hechos y de lo que los actores sociales colectivos piensan, sienten y hacen.

En relación a la evidencialidad, podemos apreciar que se construye a través de recursos de contracción dialógica y de monoglosia. La Atribución por Asimilación, esto es la mención directa o indirecta de la voz de Viqueira, sólo se considera en los primeros tres complejos clausales y al final de la respuesta del estudiante. Asimismo, se observa un constante uso del nosotros inclusivo o de usos impersonales, el primero para mostrar un acuerdo con Viqueira y se asume esa misma solidaridad con el lector —el profesor-, y el segundo para ir estableciendo los hechos y pensamientos/sentir/actuar de los actores colectivos desde una contracción dialógica, dándolos como una realidad no sujeta a discusión o con poco espacio a posiciones alternativas (se ve, vemos, veremos, comienza a verse). No se aprecia una reflexión crítica, sino sólo la reproducción de los planteamientos de Viqueira.

La evidencialidad se construye junto con una causalidad congruente mediante el uso de expresiones con procesos relacionales atributivos, con lo cual se establece que las explicaciones históricas están demostradas, que no pueden ser de otra forma y que no existe espacio para posiciones alternativas. El conector causativo/continuativo "así" permite ir construyendo una cadena causal superficial (Es así - cuatro veces-; Es de esta forma). El o la estudiante no recupera los planteamientos nucleares del autor, esto es, la respuesta se articula desde los hechos antecedentes (analfabetismo de la plebe, por ejemplo) y desde los hechos consecuentes, así como los planteamientos sobre el pensar y sentir de la elite - principalmente-y de la plebe, en menor grado (motivaciones morales y cul- 
turales). Estos planteamientos son más determinantes en la explicación histórica construida por el o la estudiante que las condiciones políticas y económicas, que prácticamente no son elaboradas en su respuesta. Las únicas referencias al factor económico y político se realizan de manera simplificada y con un lenguaje casi coloquial.

\section{Ejemplo 6:}

Viqueira realiza en su obra un cuestionamiento a la tesis clásica que decía que en S. XVIII novohispano se había producido un relajamiento de las costumbres populares. Él plantea que si bien se produce un relajamiento de la elite la que adquiere valores ilustrados y es parte de un proceso de afrancesamiento que hace que cambie el paradigma según el cual analiza la realidad social. Así, comienza a juzgar a la plebe desde la perspectiva de sus nuevos valores, basados en la moderación, la razón, el orden, la salud, etc. De esta manera, las tradiciones que habían estado instaladas en la sociedad, comienzan a ser juzgadas e intoleradas por la elite, la cual comienza a ejercer gran represión contra el pueblo.

La elite desea entonces difundir sus valores nuevos, llevar la ilustración a todo el pueblo y ejercer el control de la sociedad según estos nuevos paradigmas de conducta. Para esto, hace una reformación de las diversiones y tradiciones populares, eliminando aquellos que no le sirve para su propósito y adoptando lo que si puede serle útil.

El teatro es un gran ejemplo de esto. Éste era una tradición muy arraigada en el pueblo y que siempre había sido protegida y promovida por la elite. Sin embargo, se verá en esta diversión un peligro por los desordenes sociales, por la promiscuidad de los temas que se presentaban, por los bailes indecentes, por el comportamiento de los actores tras bambalinas, etc. La elite decide así tomar el teatro como un mecanismo de difusión de sus valores ya que una forma de representar lo que se debía ser. La gran mayoría de la población era analfabeta, por lo que era muy difícil de educar. Al no poder transmitir valores a través de la escritura, decide hacerse con la actuación.

El teatro había representado siempre un micro- cosmos de la sociedad. En este espacio se juntaban ricos y pobres y era una diversión que no distinguía por calidad o nivel social. Era así una forma fácil de llegar a todos y transmitir ideas. La elite tomó así el teatro pero lo reforma para lograr su objetivo. Se censuran los temas promiscuos y banales y se introducen temas cultos, inspirados en las luces. Se controlan las formas y comportamientos sociales que se daban en el teatro. Se impone el realismo como el único criterio válido para la representación de las obras (...)

Todas estas reformas fueron así un intento por ilustrar al pueblo, por transmitir los valores nuevos de la elite y por disciplinar a la gente. Sin embargo, había un problema que limitaba este propósito. El teatro daba ingresos importantes que la elite quería seguir recibiendo, pero el pueblo no se sentía llamado a ir a estas funciones que no comprendía porque funcionaban con otros paradigmas mentales. Los temas "cultos" no eran entendidos por la población. Si la gente dejaba de ir bajaban los ingresos. Se dio una pugna entre el interés de ilustrar con el factor económico. Había que intentar compatibilizar estos dos aspectos (...)

Podemos ver entonces cómo la elite, fruto de un cambio en sus valores, comienzan a tener una actitud de intolerancia ante prácticas que antes había aceptado. La vulgaridad, el desorden social, la promiscuidad y todas estas actitudes que se podían dar en las diversiones populares, son controladas y castigadas por la elite que intenta difundir sus nuevos valores. Así, el pueblo es reprimido por la elite, la cual sufre una relajación de su forma de ser tradicional, un cambio. No es entonces que el pueblo se relaje, sino que el paradigma desde el cual la elite juzga los comportamientos se vuelve más intolerante (ID 41).

El o la estudiante produce un relato histórico que se inicia con una fuerte estructuración desde los actores sociales (elite, plebe, pueblo) y lo que cada grupo social considera legítimo en relación a la actividad teatral. Sin embargo, hay dos grandes diferencias en relación a la respuesta más débil de este control de lectura (ver ejemplo 5): primero, que este estudiante elabora 
su relato desde las nociones que cada grupo sostiene, lo que logra a través de grupos nominalizados como un relajamiento de las costumbres populares, un relajamiento de la elite, un proceso de afrancesamiento de la realidad social. Esto permite que su respuesta se estructure desde un mayor nivel de abstracción y desde procesos sociales (ideas) más que desde lo que hacen y sienten los actores involucrados. Segundo, el relato se construye en su inicio desde valoraciones de juicios de estima y sanción social respecto de los hechos y pensamientos de uno y otro grupo (se produce un relajamiento de la elite la que adquiere valores ilustrados; comienza a juzgar a la plebe). Sin embargo, luego evoluciona a una respuesta desde la pugna entre dos sistemas de pensamiento y de los hechos concretos (económicos) que inciden en la transformación del teatro (Se dio una pugna entre el interés de ilustrar con el factor económico. Había que intentar compatibilizar estos dos aspectos), tal como se orienta la pregunta hecha por el profesor.

En relación a la construcción de la evidencialidad, el estudiante comienza reconociendo la voz de Viqueira en su discurso (heteroglosia por extravocalización de Atribución de Asimilación), pero luego tiende a expresarse de manera monoglósica con fuerte uso de procesos relacionales y materiales en cláusulas afirmativas y usos impersonales. Esta monoglosia oculta la voz de Viqueira, que sigue presente durante toda la respuesta. El o la estudiante también recurre con frecuencia a los usos de "se" impersonal (se impone, se ordena, se controlan). Este uso impersonal de procesos materiales y relacionales en cláusulas afirmativas es predominante, y sólo en tres oportunidades utiliza recursos de Proclamación de Respaldo con expresiones de evidencialidad (es un ejemplo de esto, se verá y podemos ver), construyendo el discurso desde una posición de contracción dialógica. Así, el o la estudiante presenta las ideas de Viqueira como 'verdades' del proceso histórico tratado. Además, al igual que el estudiante de rendimiento más dé- bil, recurre a la conjunción causativa "así" en cinco oportunidades, de manera que se combinan (coupling) los significados de evidencialidad y causalidad. El estudiante utiliza esta conjunción para reafirmar el argumento de Viqueira y como un recurso adicional para la construcción de la evidencialidad.

El o la estudiante jerarquiza la evidencia deteniéndose principalmente en lo que en Viqueira es planteado como las condiciones económicas, sociales y culturales. Asimismo, organiza su argumento en torno a las condiciones morales (sociales) planteadas por este autor. Sin embargo, las condiciones políticas están prácticamente ausentes (reformas borbónicas), así como las motivaciones políticas (control de la Corona Española de la Nueva España). El o la estudiante considera, eso sí, de forma medular las condiciones económicas (falta de financiamiento del teatro por la incorporación de los valores ilustrados en sus temáticas y formas de representación), y logra articular su respuesta desde las tesis principales o nucleares de Viqueira y ejemplificar con hechos que funcionan como antecedentes y consecuentes (creación de nuevos teatros; cambio de actitud del pueblo pero permanencia de sus gustos). Algunos hechos mencionados podrían, tal vez, considerarse como situaciones/ motivaciones sociales y culturales de la nueva elite, las cuales constituyen parte importante de las tesis nucleares del autor.

\section{Conclusiones}

El discurso de la historia y el discurso recontextualizado construido por los estudiantes universitarios pertenecen a un dominio de conocimiento que descansa en recursos específicos de construcción de significados. El análisis realizado nos ha permitido determinar que tanto el propósito de las preguntas de los controles de lectura como la evidencia seleccionada para ellos dan el contexto para la construcción de la evidencialidad y para la incorporación de los recursos lingüísticos utilizados por los estudian- 
tes. La consigna orienta la respuesta, ya que el propósito de la pregunta y, por lo mismo, la construcción discursiva de los y las estudiantes quedan subordinados al planteamiento de la misma, lo que enmarca las posibilidades de elaboración de significación histórica. Así, la inclusión o exclusión de hechos, condiciones, motivaciones, o la articulación de los mismos en planteamientos o ideas nucleares de los autores de fuentes primarias y bibliografía secundaria está forzada desde la consigna. Sin embargo, hemos constatado en el análisis de las respuestas de los y las estudiantes que los mecanismos de inclusión o de exclusión varían y que pueden representar diferentes grados de calidad de incorporación de la evidencia en la construcción de explicaciones históricas.

En el primer caso, que trata de una crónica que expone hechos en una secuencia temporal, y en las respuestas se mencionan algunos de los hechos indicados en la fuente primaria, considerados por los estudiantes como garantías evidenciales para elaborar su relato. En ambos ejemplos, así como en el resto del corpus analizado para este nivel, se incorporan elementos que no están presentes en la fuente (conceptos teóricos, crítica a otras fuentes), al parecer para intentar construir explicación. Esta presentación de hechos como "evidentes" y "necesarios" (sin explicación cabal de por qué ocurrió lo que ocurrió, es decir, sin que se incluyan motivaciones y condiciones con los hechos) puede configurarse como una estrategia del estudiante para darle claves al lector/corrector. En términos de evidencialidad, esta estrategia se muestra tanto en heteroglosia (intravocalización contracción dialógica, ejemplo 1) como en monoglosia (cláusulas impersonales, ejemplo 2). La estrategia no es exclusiva de la heteroglosia o de la monoglosia, sino que de la ausencia de perspectiva del estudiante sobre la evidencia y la incorporación fundamentalmente de hechos en la respuesta (pocas motivaciones y prácticamente ausencia de condiciones). En el segundo y tercer caso, en los que los y las estudiantes debían incorporar bibliografía secundaria en sus respuestas, la extravocalización por Atribución de Asimilación, así como el uso de recursos de contracción dialógica y monoglosia, también se utilizan de manera estratégica como una manera de "dar guiños" al lector/corrector, sin que necesariamente se introduzcan adecuadamente los planteamientos de los autores. En sus repuestas se destacan, asimismo, los recursos de contracción dialógica, que son empleados por los y las estudiantes para construirse a sí mismos/as como 'expertos/ as' historiadores/as y demostrar que manejan las fuentes asignadas por el o la profesor/a del curso (se verá, era así, fueron así, podemos ver), recursos que combinan los significados de evidencialidad y causalidad.

De esta manera, los resultados de esta investigación arrojaron tres tendencias predominantes presentes en las respuestas del género "control de lectura de Historia" de los estudiantes: a) la utilización estratégica de heteroglosia por intravocalización de contracción dialógica, la cual es utilizada como un mecanismo para indicar al evaluador el conocimiento de los hechos expuestos en la fuente primaria, sin generar algún tipo de explicación histórica más elaborada; b) la utilización de heteroglosia por extravocalización de Atribución por Asimilación, sin que esto implique que la incorporación de la evidencia sea de buena calidad, ya que en muchos casos se incorpora la evidencia de manera errónea o estratégica de los hechos; c) la utilización de recursos de contracción dialógica y recursos de orientación monoglósica en los que se oculta la voz del autor.

Finalmente, se concluye que los mecanismos de construcción de la dimensión de evidencialidad predominantes están directamente vinculados al tipo de evidencia utilizada por los estudiantes y profesores. La discusión establece la necesidad de incorporar en la formación de los historiadores esta triangulación: el tipo de evidencia seleccionada (fuentes primarias, 
bibliografía secundaria), la consigna del género académico "control de lectura de Historia” y el desarrollo de las etapas del género (tanto control de lectura como otros: ensayo, monografía, tesis). En dicha triangulación se desarrollan las orientaciones de la evidencialidad como parte de la construcción de la significación histórica.

Asimismo, consideramos que la alfabetización académica disciplinar permitirá que los estudiantes de Historia, muchos de los cuales serán futuros profesores, comprendan las formas en las que se construye el discurso y la significación histórica, manejando los recursos lingüísticos para construir evidencialidad dependiendo del nivel de la carrera que se encuentren cursando. De esa manera, al incorporar la evidencia, el estudiante debe manejar recursos de codificación de la intersubjetividad, así como recursos de negociación de significados ideacionales, que le permitan construir perspectiva propia.

\section{Bibliografía citada}

Achugar, Mariana, 2011: "Aproximaciones discursivas a la transmisión del pasado reciente" en Teresa Oteiza y Derrin Pinto (eds.): En (re)construcción: discurso, identidad y nación en los manuales escolares de historia y de ciencias sociales, Santiago: Editorial Cuarto Propio, 43-87.

Bernsteln, Basil, 2000: Pedagogy, Symbolic Control and Identity. Theory, research, critique, New York, Oxford: Rowman \& Littlefield Publishers.

Coffin, Caroline, 2006: Historical discourse: the language of time, cause and evaluation, London, New York: Continuum.

FalRclough, Norman, 2003: Analysing Discourse. Textual analysis for social research, London, New York: Routledge.

Fontana, Josep, 1992: La historia después del fin de la historia. Reflexiones acerca de la situación actual de la ciencia histórica, Barcelona: Crítica.
Fuller, Gillian, 1995: Engaging Culture: Negotiating Discourse in Popular Science. Thesis submitted in fulfilment of the requirements for the degree of Doctor of Philosophy, Semiotic Programme, University of Sydney.

Giudice, Jacqueline y Estela Moyano, 2011: "Género y formación de ciudadanos: la re-construcción del período 1976-1983 en manuales argentinos para la escuela primaria" en Teresa Oteiza y Derrin Pinto (eds.): En (re)construcción: Discurso, identidad y nación en los manuales escolares de historia y de ciencias sociales, Santiago: Cuarto Propio, 205-268.

GiUdice, Jacqueline y Estela Moyano, 2013: "El período 76-83 en la Argentina: género, ideología y formación de ciudadanos en manuales de Ciencias Sociales" en Estela Moyano (coord.): Aprender ciencias y humanidades: una cuestión de lectura y escritura. La mediación del lenguaje escrito y otros sistemas semióticos en la enseñanza y el aprendizaje de las ciencias en la escuela, Los Polvorines: Universidad Nacional de General Sarmiento, 399-426.

Halliday, Michael, 1994: An Introduction to Functional Grammar, London: Edward Arnold.

Henríuez, Rodrigo y Marcela Ruiz, 2014: "Chilean students learn to think historically: Construction of historical causation through the use of evidence in writing", Linguistics and Education 25, 145-157.

Henríuez, Rodrigo y Valentina Canelo, 2014: "Géneros históricos y construcción de la significación histórica en estudiantes de Licenciatura en Historia”, Onomázein, número especial IX ALSFAL (2014).

Lemke, Jay, 1995: Textual Politics: Discourse and Social Dynamics, London: Taylor \& Francis.

Manghi, Dominique, 2013a: "Géneros en la enseñanza escolar: configuraciones de significado en clases de historia y biología desde una perspec- 
tiva multimodal", Revista Signos 82 (46), 236-247. Manghi, Dominique, 2013b: "Representación y comunicación del conocimiento en Educación Media: análisis multimodal del discurso de materiales utilizados para la enseñanza escolar de la historia y de la biología”, Onomázein 27, 25-52.

Martin, Jim, 2002: "Writing History: Construing Time and Value in Discourses of the Past" en Mary Schleppegrell y Cecilia Colombi (eds.): Developing Advanced Literacy in First and Second Languages: Meaning with Power, Mahwah, NJ: Lawrence Erlbaum Associates.

MarTin, Jim y David Rose, 2007: Working with Discourse. Meaning beyond the clause, segunda edición, London: Continuum.

Martin, Jim y David Rose, 2008: Genre Relations: mapping culture, London: Equinox.

Martin, Jim y Peter White, 2005: The Language of Evaluation. Appraisal in English, New York: Palgrave Macmillan.

MaRTIN, Jim y Ruth Wodak (eds.), 2003: Re/reading the Past. Critical and functional perspectives on time and value, Amsterdam/Philadelphia: John Benjamins Publishing Company.

Maton, Karl, Jim Martin y Erika Matruglio, 2010: "Historical cosmologies: Epistemology and axiology in Australian secondary school history discourse", Revista Signos 43 (74), 433-463.

Maton, Karl, 2014: Knowledge and Knowers. Towards a realist sociology of education, London/New York: Routledge.

Moss, Gillian 2010: "Textbook language, ideology, and citizenship", Functions of Language 17 (1), 71-93.

Moss, Gillian, Norma Barletta, Diana Chamorro y Jorge Mizuno, 2013: "La metáfora gramatical en los textos escolares de Ciencias Sociales en español”, Onomázein 28, 88-104.
Morano, Estela, 2010: "Aportes del análisis de género y discurso a los procesos de enseñanza y aprendizaje escolar: las ciencias biológicas y la historia", Discurso y Sociedad 4 (2), 294-331.

Oteiza, Teresa, 2006: El discurso pedagógico de la historia. Un análisis lingüístico sobre la construcción ideológica de la historia de Chile (19702001), Santiago: Frasis editores.

OteízA, Teresa, 2009: "Solidaridad ideológica en el discurso de la historia: tensión entre orientaciones monoglósicas y heteroglósicas", Revista Signos. Estudios de Lingüística 42 (70), 219-244.

OteízA, Teresa, 2011: "Representación de las memorias del pasado: intersubjetividad en el discurso pedagógico de la historia" en Teresa Oteiza y Derrin Pinto (eds.): En (re)construcción: Discurso, identidad y nación en los manuales escolares de historia y de ciencias sociales, Santiago: Editorial Cuarto Propio, 129-172.

Oteíza, Teresa, 2013: "Recontextualización del pasado nacional reciente en los manuales escolares de historia" en Estela Moyano (coord.): Aprender ciencias y humanidades: una cuestión de lectura y escritura. La mediación del lenguaje escrito y otros sistemas semióticos en la enseñanza y el aprendizaje de las ciencias en la escuela, Los Polvorines: Universidad Nacional de General Sarmiento, 367-398.

OteízA, Teresa, 2014: "Intertextualidad en la recontextualización pedagógica del pasado reciente chileno", Discurso \& Sociedad 8 (1), 109-136.

OteízA, Teresa y Claudio Pinuer, 2012: "Prosodia valorativa: construcción de eventos y procesos en el discurso de la historia", Discurso y Sociedad 6 (2), 418-446.

Oteíza, Teresa y Claudio Pinuer, 2013: "Valorative Prosody and the symbolic construction of time in historical recent national discourses", Discourse Studies 15 (1), 43-64.

PAXTON, Richard, 2002: "The influence of author visibility on high school students solving a historical problem", Cognition and Instruction 20 (2), 197-248. 
Pinto, Derrin, 2011: "La cortesía y formación del espíritu nacional en libros escolares franquistas" en Teresa Oteiza y Derrin Pinto (eds.): En (re) construcción: Discurso, identidad y nación en los manuales escolares de historia y de ciencias sociales, Santiago: Cuarto Propio, 173-204.

Ricoeur, Paul, 2010: La memoria, la historia y el olvido, México: Fondo de Cultura Económica.

Schleppegrell, Mary, 2004: The Language of Schooling. A Functional Linguistics Perspective, London: Routledge.

Schleppegrell, Mary, Mariana Achugar y Teresa OteiZA, 2004: "The Grammar of History: Enhancing Content-Based Instruction through a Functional Focus on Language", Tesol Quarterly 38, 67-93.

Stahl, Steven y Cynthia Shanahan, 2004: "Learning to think like a historian. Disciplinary knowledge through critical analysis of multiple documents" en Tamara Jetron y Janice Dole (eds.): Adolescent Iiteracy research and practice, New York: Guilford Press, 94-115.

Troulllot, Michael, 1995: Silencing the Past. Power and the Production of History, Boston: Beacon Press.

VeEL, Robert y Caroline Coffin, 1996: "Learning to Think Like a Historian: The Language of Secondary School History" en Ruqaiya HASAN y Geoff Willıams (eds.): Literacy in Society, London: Longman, 191-231.

WhITE, Peter, 2000: The appraisal website [http:// www.grammatics.com/appraisal/, last update: March, 5th, 2005].

White, Peter, 2003: "Beyond modality and hedging: A dialogic view of the language of intersubjective stance", Text 23 (2), 259-284.

\subsection{Fuentes primarias y bibliografía secundaria de los controles de lectura}

Chust Calero, Manuel, 2003: "La coyuntura de la crisis: España, América” en Germán Damas (dir.):
Historia General de América Latina: La crisis estructural de las sociedades implantadas, París: Ediciones UNESCO, Editorial Trotta, 55-90.

Guerrero, Gonzalo, s. f.: El encuentro con el mundo maya. La relación de Gonzalo Guerrero.

GuerRa, François-Xavier, 1992: Modernidad e independencias. Ensayos sobre las revoluciones hispánicas, Madrid: Fondo de Cultura Económica.

VIQueIRA, Juan Pedro, 1987: ¿Relajados o reprimidos? Diversiones públicas y vida social en la ciudad de México durante el Siglo de las Luces, Ciudad de México: Fondo de Cultura Económica. 